\title{
Discovery of collimated ejection from the symbiotic binary BF Cygni ${ }^{\star}$
}

\author{
A. Skopal ${ }^{1}$, N. A. Tomov ${ }^{2, \star \star}$, and M. T. Tomova ${ }^{2}$ \\ 1 Astronomical Institute, Slovak Academy of Sciences, 05960 Tatranská Lomnica, Slovakia \\ e-mail: skopal@astro.sk \\ 2 Institute of Astronomy and NAO, Bulgarian Academy of Sciences, PO Box 136, 4700 Smolyan, Bulgaria
}

Received 2 January 2013 / Accepted 29 January 2013

ABSTRACT

\begin{abstract}
Context. Detection of collimated ejection from white dwarfs (WD) in symbiotic binaries is very rare and has employed a variety of methods in X-ray, radio, optical imagery, and spectroscopy. To date, its signature in the optical spectra has only been recorded for four objects (MWC 560, Hen 3-1341, StH $\alpha$ 190, and Z And).

Aims. We present the first observational evidence of highly-collimated bipolar ejection from the symbiotic binary BF Cyg, which developed during its current (2006-12) active phase, and determine their physical parameters.

Methods. We monitored the outburst with the optical high-resolution spectroscopy and multicolour $U B V R_{\mathrm{C}} I_{\mathrm{C}}$ photometry.

Results. During 2009, three years after the 2006-eruption of BF Cyg, satellite components to $\mathrm{H} \alpha$ and $\mathrm{H} \beta$ lines emerged in the spectrum. During 2012, they became stable and were located symmetrically with respect to the main emission core of the line. Spectral properties of these components suggest bipolar ejection collimated within an opening angle of $\lesssim 15^{\circ}$, whose radiation is produced by an optically thin medium with the emission measure of $1-2 \times 10^{59}(d / 3.8 \mathrm{kpc})^{2} \mathrm{~cm}^{-3}$.

Conclusions. Formation of the collimated ejection a few years after the eruption and its evolution on a time scale of years at a constant optical brightness can aid us in better understanding the accretion process during the active phases of symbiotic stars.
\end{abstract}

Key words. ISM: jets and outflows - binaries: symbiotic - stars: individual: BF Cygni

\section{Introduction}

BF Cyg is an eclipsing symbiotic binary (Skopal 1992; Pereira \& Landaberry 1996) comprising an M5 III giant (Mürset \& Schmid 1999) and a white dwarf (WD) on a 757.2-d orbit (e.g. Fekel et al. 2001). The historical light curve of BF Cyg is dominated by a symbiotic nova-like outburst, which occurred around 1895 (Jacchia 1941) with a slow decline to the pre-outburst magnitudes, for almost one century, and a number of Z And-type outbursts superposed on the nova-like profile (see Fig. 1 of Skopal et al. 1997; Leibowitz \& Formiggini 2006; Siviero et al. 2012). During outbursts the emission line spectrum is represented by the lines of low ionized elements (H I, He I, and neutral or singly ionized metals), whose profiles are often P Cyg-type. Their absorption component indicates a mass outflow at 100-200 to $500 \mathrm{~km} \mathrm{~s}^{-1}$ from the hot star (González-Riestra et al. 1990; Cassatella et al. 1992; Skopal et al. 1997; McKeever et al. 2011).

The recent outburst of BF Cyg started on 2006 July 31 (Munari et al. 2006), when the star's brightness increased from $U \sim 11.1$ to $U \sim 9.3$ during 2006 August (Skopal et al. 2007). The low ionization state of the emission line spectrum and development of P Cyg profiles of H I, He I, and some Fe II lines were reported by Sitko et al. (2006), Iijima (2006), and McKeever et al. (2011). An interesting feature of the current active phase is a high level of the star's brightness, which is kept

\footnotetext{
* Based on data collected with 2-m telescope at the Rozhen National Astronomical Observatory and the David Dunlap Observatory.

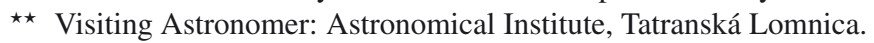

continuously at $U \lesssim 10$ from the 2006 eruption to the present, i.e. for $>6.3$ years (Skopal et al. 2012, Fig. 1 here).

Our spectroscopic monitoring of BF Cyg revealed for the first time the emergence of satellite-emission components to the $\mathrm{H} \alpha$ and $\mathrm{H} \beta$ emission lines from 2009. To date, similar emissions in the optical spectra of symbiotic stars have only been discovered for Hen 3-1341 (Tomov et al. 2000), StH $\alpha 190$ (Munari et al. 2001), and Z And (Skopal \& Pribulla 2006), although a few jets have been discovered by other methods. In this contribution we present the evolution of these jet features from their emergence in 2009 to 2012 and determine their basic parameters.

\section{Observations and data reduction}

Our spectroscopic observations were carried out at the David Dunlap Observatory, University of Toronto (DDO) and the Rozhen National Astronomical Observatory (RNAO). At the DDO the high-resolution spectroscopy was performed by the single dispersion slit spectrograph equipped with a Jobin Yovon Horiba CCD detector $(2048 \times 512$ pixels of $13.5 \mu \mathrm{m}$ size; thinned back illuminated chip) mounted at the Cassegrain focus of the $1.88-\mathrm{m}$ telescope. The resolution power was 12000 and 8000 around the $\mathrm{H} \alpha$ and $\mathrm{He} \mathrm{II} 4686-\mathrm{H} \beta$ regions, respectively. During each night two very different exposures were applied to obtain a well-defined $\mathrm{H} \alpha$ profile and the continuum.

At the RNAO the high-resolution spectroscopy was carried out by the single dispersion slit spectrograph equipped with the Photometrics CCD camera $(1024 \times 1024$ pixels of $24 \mu \mathrm{m}$ size $)$ mounted at the coudé focus of the 2-m RCC telescope. The 

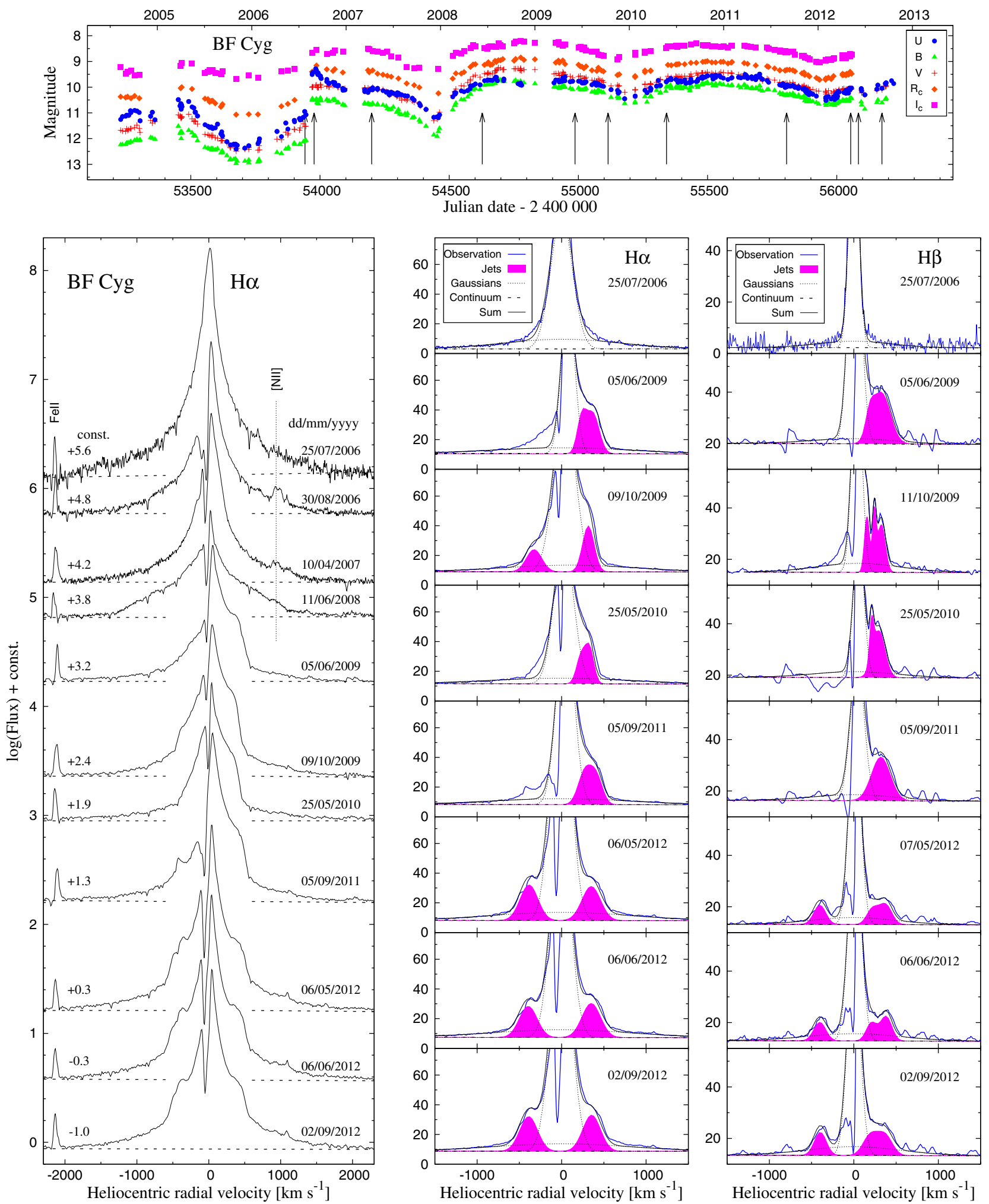

Fig. 1. Top panel: $U B V R_{\mathrm{C}} I_{\mathrm{C}}$ light curves of BF Cyg covering its current, 2006-12, active phase (Skopal et al. 2007, 2012, and our unpublished data for $>2011.9)$. Arrows indicate times of our spectroscopic observations. Lower panels: evolution of the $\mathrm{H} \alpha$ and $\mathrm{H} \beta$ line profiles along the outburst. The filled curves represent the jet emission components (Sect. 3.2). Fluxes are in $10^{-13} \mathrm{erg} \mathrm{cm}^{-2} \mathrm{~s}^{-1} \AA^{-1}$.

resolution power was 32000 and 24000 around the $\mathrm{H} \alpha$ and $\mathrm{H} \beta$ regions, respectively. To obtain a better signal-to-noise ratio, two exposures per night were made in some cases.

Basic treatment of the spectra was done using the IRAFpackage software. The spectra were converted to fluxes

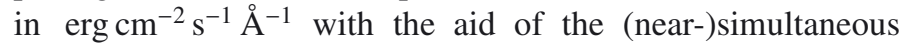

$B V R_{\mathrm{C}} I_{\mathrm{C}}$ photometry corrected for emission lines. Uncertainties of such a continuum calibration are a few percent for the star's brightness around 9 mag in the $V R$ passbands (see Skopal 2007, in detail). Observations were dereddened with $E_{B-V}=0.35$ (Mürset et al. 1991). The journal of spectroscopic observations is given in Table 1. 
Table 1. Log of spectroscopic observations.

\begin{tabular}{lcccc}
\hline \hline $\begin{array}{l}\text { Date } \\
\text { dd/mm/yyyy }\end{array}$ & $\begin{array}{c}\text { Julian date } \\
\text { JD }-2400000\end{array}$ & $\begin{array}{c}\text { Region } \\
{[\mathrm{nm}]}\end{array}$ & $\begin{array}{c}T_{\text {exp }} \\
{[\mathrm{min}]}\end{array}$ & Observatory \\
\hline $25 / 07 / 2006$ & 53941.843 & $642-671$ & 10 & DDO \\
& 53941.857 & $462-492$ & $1+5$ & DDO \\
$30 / 08 / 2006$ & 53977.621 & $642-671$ & $2+10$ & DDO \\
& 53977.602 & $462-492$ & $2+20$ & DDO \\
$10 / 04 / 2007$ & 54200.860 & $642-671$ & $1+15$ & DDO \\
& 54200.908 & $462-492$ & 20 & DDO \\
$11 / 06 / 2008$ & 54628.828 & $642-671$ & $2+30$ & DDO \\
& 54628.801 & $462-492$ & $2+30$ & DDO \\
$05 / 06 / 2009$ & 54988.461 & $649-670$ & 20 & RNAO \\
& 54988.496 & $478-498$ & 20 & RNAO \\
$09 / 10 / 2009$ & 55114.289 & $649-670$ & 15 & RNAO \\
$11 / 10 / 2009$ & 55116.248 & $478-498$ & 20 & RNAO \\
$25 / 05 / 2010$ & 55342.420 & $647-667$ & 20 & RNAO \\
& 55342.467 & $478-498$ & $2 \times 20$ & RNAO \\
$05 / 09 / 2011$ & 55810.300 & $647-667$ & 20 & RNAO \\
& 55810.318 & $478-498$ & 20 & RNAO \\
$06 / 05 / 2012$ & 56054.470 & $647-667$ & 20 & RNAO \\
$07 / 05 / 2012$ & 56054.517 & $478-498$ & $2 \times 20$ & RNAO \\
$06 / 06 / 2012$ & 56085.461 & $647-667$ & 20 & RNAO \\
& 56085.480 & $478-498$ & 20 & RNAO \\
$02 / 09 / 2012$ & 56173.276 & $642-662$ & 20 & RNAO \\
& 56173.254 & $473-493$ & 20 & RNAO \\
\hline
\end{tabular}

\section{Results}

\subsection{Evolution in the $\mathrm{H} \alpha$ and $H \beta$ lines}

Figures 1 and 2 show evolution of the $\mathrm{H} \alpha$ and $\mathrm{H} \beta$ profiles from the 2006 brightening to 2012 September. The broad wings expanding to $\sim \pm 2000$ in $\mathrm{H} \alpha$ and $\sim \pm 1000 \mathrm{~km} \mathrm{~s}^{-1}$ in $\mathrm{H} \beta$, were present in all spectra. Significant variations were observed mainly at/around the line cores. We summarize them as follows.

(i) Just prior to the main brightening in 2006 August (2006 July 25 spectrum), the profiles showed a single emission peak with the broad wings.

(ii) During the 2006 eruption, a sharp absorption developed on the blue side of the profile throughout the whole active phase. At some times, the $\mathrm{H} \beta$ profile was P Cyg type.

(iii) During 2009, additional satellite emission bumps appeared on the sides of the $\mathrm{H} \alpha$ and $\mathrm{H} \beta$ emission core, placed at a few times $100 \mathrm{~km} \mathrm{~s}^{-1}$ from the line center (Table 2).

(iv) During 2009-2011, the red satellite emissions were more pronounced, because of a strong absorption from the blue side of the line, which often consumed the blue $\mathrm{H} \beta$ wing totally. During 2010 May-2012 May, the blue $\mathrm{H} \beta$ satellite component transformed from absorption into emission.

(v) During 2012, the satellite emissions occurred on both sides of the $\mathrm{H} \alpha$ and $\mathrm{H} \beta$ lines, nearly symmetrically with respect to their central emission (the dotted line in Fig. 1).

\subsection{Parameters of the satellite components}

To isolate the satellite components from the whole line profile, we formally fitted the emission line core and its extended foot component with the Gaussian function (dotted lines in Fig. 1). We assumed them to be symmetrical with respect to the reference wavelength as suggested by observations during 2012 and before the eruption. Then the residual emissions were fitted with additional Gaussians (Fig. 1). From their parameters (the central wavelength, maximum $I$, and the width $\sigma$ ), we derived

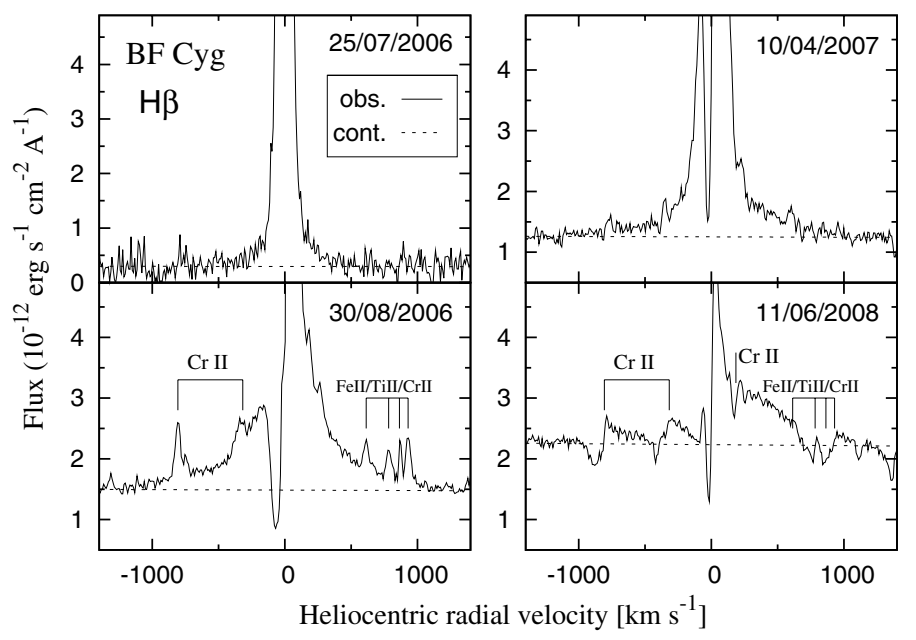

Fig. 2. Evolution in the $\mathrm{H} \beta$ line profile from around the 2006 August eruption to the formation of jets in 2009 (see Fig. 1), showing the pronounced absorption in P Cyg profiles of low-ionized metal lines at the spectrum from 11/06/2008, prior to detecting jets in 2009 .

their radial velocity, $R V_{\mathrm{S}}$, flux $F_{\mathrm{S}}=\sqrt{2 \pi} I \sigma$ and the width $F W H M_{\mathrm{S}}=2 \sqrt{2 \ln (2)} \sigma$. If more Gaussians were used to fit the satellite component, the above-mentioned parameters were estimated from their sum. Finally, we corrected the heliocentric radial velocity $R V_{\mathrm{S}}$ for the systemic velocity and the orbital motion of the hot component as given by elements of Fekel et al. (2001). Resulting parameters are introduced in Table 2. This approach and the resolution of our spectra allowed us to estimate uncertainties in the $R V_{\mathrm{S}}$ to $10-20 \mathrm{~km} \mathrm{~s}^{-1}$, in the $F W H M_{\mathrm{S}}$ to $0.4 \AA$ and in fluxes to $10-20 \%$ of the observed values.

The relatively small width of the satellite components $\left(F W H M_{\mathrm{S}}=245 \pm 11 \mathrm{~km} \mathrm{~s}^{-1}\right.$; the average from all values in Table 2 and the rms error) and their position $R V_{\mathrm{S}}$ in the line profile suggest that these emissions are produced by radiation of a highly collimated ejection by the central star.

\subsection{Opening angle of the jets}

The narrow profile of satellite lines with often sharp edges and a flat top (see Fig. 1) allows us to assume a constant velocity of the emitting particles in the jet. Then the observed line width can be the result of the dispersion in the line-of-sight velocity components of the jet particles. This can be fulfilled if the jet emitting medium has the geometry of a narrow cone with the peak at the central object and it is characterized by a small opening angle $\theta_{0}$. Assuming that the jets were launched perpendicularly to the disk plane that coincides with the orbital one, the opening angle can be expressed as a function of the observed width of the satellite component $H W Z I_{\mathrm{S}}, R V_{\mathrm{S}}$ and the orbital inclination $i$ as

$\theta_{0}=2 \sin ^{-1}\left[\frac{H W Z I_{\mathrm{S}}}{R V_{\mathrm{S}} \tan (i)}\right]$,

where $H W Z I_{\mathrm{S}}$ can be approximated with $F W H M_{\mathrm{S}}$ (see Skopal et al. 2009, in detail). The orbital inclination is high, because of eclipses measured in both the optical and far-UV continuum (see Sect. 1). Skopal et al. (1997) estimated $i \approx 70^{\circ}-90^{\circ}$. For the purpose of this paper, we adopted $i=80^{\circ}$. Such a high $i$ is consistent with the mass function of $0.0239 M_{\odot}$, the mass ratio of $\sim 3.8$, and the considered mass of the WD, $M_{\mathrm{WD}}=0.6 M_{\odot}$ (Fekel et al. 2001). Corresponding parameters from Table 2 yield the average value of $\theta_{0}=15^{\circ} .2 \pm 1.5 \times \Delta i$, where the uncertainty represents 
Table 2. Parameters of Gaussian fits to the satellite emissions in $\mathrm{H} \alpha$ and $\mathrm{H} \beta$ lines.

\begin{tabular}{lccccccccccc}
\hline \hline $\begin{array}{l}\text { Date } \\
\text { dd/mm/yyyy }\end{array}$ & Line & \multicolumn{2}{c}{$R V_{\mathrm{S}}$} & \multicolumn{3}{c}{$F W H M_{\mathrm{S}}$} & \multicolumn{3}{c}{$\theta_{0}$} & \multicolumn{3}{c}{$F_{\mathrm{S}}$} & \multicolumn{3}{c}{$E M_{\text {jet }}$} \\
& & $S^{-}$ & $S^{+}$ & $S^{-}$ & $S^{+}$ & $S^{-}$ & $S^{+}$ & $S^{-}$ & $S^{+}$ & $S^{-}$ & $S^{+}$ \\
\hline $05 / 06 / 2009$ & $\mathrm{H} \alpha$ & - & +298 & - & 270 & - & 18.4 & - & 18.0 & - & 17 \\
& $\mathrm{H} \beta$ & - & +295 & - & 302 & - & 20.8 & - & 10.1 & - & 25 \\
$09 / 10 / 2009$ & $\mathrm{H} \alpha$ & -334 & +308 & 215 & 161 & 13.0 & 10.6 & 7.5 & 11.7 & 7.1 & 11 \\
$11 / 10 / 2009$ & $\mathrm{H} \beta$ & - & +221 & - & 249 & - & 22.9 & - & 8.2 & - & 21 \\
$25 / 05 / 2010$ & $\mathrm{H} \alpha$ & - & +332 & - & 203 & - & 12.4 & - & 12.1 & - & 11 \\
& $\mathrm{H} \beta$ & - & +291 & - & 192 & - & 13.4 & - & 6.9 & - & 17 \\
$05 / 09 / 2011$ & $\mathrm{H} \alpha$ & - & +317 & - & 274 & - & 17.5 & - & 16.7 & - & 16 \\
& $\mathrm{H} \beta$ & - & +301 & - & 261 & - & 17.6 & - & 7.7 & - & 20 \\
$06 / 05 / 2012$ & $\mathrm{H} \alpha$ & -361 & +375 & 248 & 258 & 13.9 & 13.9 & 13.8 & 13.8 & 13 & 13 \\
$07 / 05 / 2012$ & $\mathrm{H} \beta$ & -378 & +334 & 167 & 302 & 8.9 & 18.3 & 2.2 & 4.0 & 5.6 & 10 \\
$06 / 06 / 2012$ & $\mathrm{H} \alpha$ & -368 & +377 & 248 & 258 & 13.6 & 13.9 & 12.1 & 13.8 & 11 & 13 \\
& $\mathrm{H} \beta$ & -376 & +333 & 167 & 319 & 9.0 & 19.4 & 2.1 & 4.4 & 5.3 & 11 \\
$02 / 09 / 2012$ & $\mathrm{H} \alpha$ & -363 & +381 & 247 & 247 & 13.8 & 13.1 & 13.5 & 14.1 & 13 & 13 \\
& $\mathrm{H} \beta$ & -370 & +307 & 214 & 344 & 11.7 & 22.7 & 2.6 & 5.5 & 6.6 & 14 \\
\hline
\end{tabular}

Notes. Radial velocity $R V_{\mathrm{S}}\left[\mathrm{km} \mathrm{s}^{-1}\right], F W H M_{\mathrm{S}}\left[\mathrm{km} \mathrm{s}^{-1}\right]$, opening angle $\theta_{0}\left[^{\circ}\right]$, flux $F_{\mathrm{S}}\left[10^{-12} \mathrm{erg} \mathrm{cm}^{-2} \mathrm{~s}^{-1}\right]$, and emission measure $E M_{\text {jet }}\left[10^{58} \mathrm{~cm}^{-3}\right]$.

a total differential of the function (1) for the uncertainty in the orbital inclination, $\Delta i$.

However, if the width of satellite components is in part caused by the velocity dispersion along the jet, then the opening angle will be $<\theta_{0}$.

\subsection{Emission measure of the jets}

Assuming that the radiation of jets is generated by the recombination transition in a line $l$ (see Skopal et al. 2009), the luminosity $L_{\text {jet }}(l)$ is related to the line emissivity, $\varepsilon_{l} n_{\mathrm{e}} n_{\mathrm{p}}$ by

$L_{\mathrm{jet}}(l)=\varepsilon_{l} \int_{V_{\mathrm{jet}}} n_{\mathrm{e}} n_{\mathrm{p}} \mathrm{d} V=\varepsilon_{l} \times E M_{\text {jet }}$,

where $\varepsilon_{l}$ is the volume emission coefficient of the line transition, $n_{\mathrm{e}}$ and $n_{\mathrm{p}}$ are the concentrations of electrons and protons, $V_{\text {jet }}$ and $E M_{\text {jet }}$ are the volume and emission measures of the jets. Thus, neglecting dependencies of $\varepsilon_{l}$ on the electron temperature and density means $E M_{\text {jet }}$ can be expressed as

$E M_{\mathrm{jet}}=4 \pi d^{2} \frac{F_{\mathrm{S}}}{\varepsilon_{l}}$.

The last column of Table 2 introduces quantities of $E M_{\text {jet }}$ for jet fluxes $F_{\mathrm{S}}$, distance $d=3.8 \mathrm{kpc}$ (Skopal 2005), and coefficients $\varepsilon_{\alpha}=1.83$ for $\mathrm{H} \alpha$ and $\varepsilon_{\beta}=0.682 \times 10^{-25} \mathrm{erg} \mathrm{cm}^{3} \mathrm{~s}^{-1}$ for $\mathrm{H} \beta$ $\left(T_{\mathrm{e}}=2 \times 10^{4} \mathrm{~K}\right.$, e.g. Hummer \& Storey 1987).

\section{Conclusion}

In this paper we reported a discovery of collimated ejection from the symbiotic star BF Cyg. The jets were indicated in the spectrum as satellite emission components to $\mathrm{H} \alpha$ and $\mathrm{H} \beta$ lines, which developed from 2009 - three years after the optical eruption. During 2012, they became bipolar (see Fig. 1). Parameters of satellite components suggest that the jets were collimated into the opening angle of $\lesssim 15^{\circ}$, and their radiation was produced by an optically thin medium with the emission measure of $\sim 1-2 \times 10^{59}(d / 3.8 \mathrm{kpc})^{2} \mathrm{~cm}^{-3}$ (Table 2).
A detailed analysis of the present, unusually long-lasting outburst should aid us in better understanding the accretion process during active phases of symbiotic stars leading to formation of collimated ejection from their WDs.

Acknowledgements. We thank the referee, Joy Nichols, for constructive comments, Theodor Pribulla for acquiring the spectra at the David Dunlap observatory, Sergei Shugarov and Matej Sekeráš for taking some photometric observations from 2012, and Zuzana Cariková for help with their treatment. This research was supported by the Slovak-Bulgarian Research and Development Cooperation project SK-BG-0015-10, by the grant BSTC No. 01/14 BulgariaSlovakia, by the grant DO 02-85 of Bulgarian Scientific Research Fund, and by a grant of the Slovak Academy of Sciences VEGA No. 2/0002/13.

\section{References}

Cassatella, A., Fernandez-Castro, T., Gonzalez-Riestra, R., \& Fuensalida, J. J. 1992, A\&A, 258, 368

Fekel, F. C., Hinkle, K. H., Joyce, R. R., \& Skrutskie, M. 2001, AJ, 121, 2219 González-Riestra, R., Cassatella, A., \& Fernández-Castro, T. 1990, A\&A, 237, 385

Hummer, D. G., \& Storey, P. J. 1987, MNRAS, 224, 801

Leibowitz, E. M., \& Formiggini, L. 2006, MNRAS, 366, 675

Iijima, T. 2006, CBET, 633

Jacchia, L. 1941, Bull. Harv. Coll. Obs., 915, 17

McKeever, J., Lutz, J., Wallerstein, G., Munari, U., \& Siviero, A. 2011, PASP, 123,1062

Munari, U., Tomov, T., Yudin, B. F., et al. 2001, A\&A, 369, L1

Munari, U., Siviero, A., Moretti, S., et al. 2006, CBET, 596

Mürset, U., \& Schmid, H. M. 1999, A\&AS, 137, 473

Mürset, U., Nussbaumer, H., Schmid, H. M., \& Vogel, M. 1991, A\&A, 248, 458

Pereira, C. B., \& Landaberry, S. J. C. 1996, AJ, 111, 1329

Sitko, M. L., Lynch, D. K., Russell, R. W., \& Venturini, C. C. 2006, IAUC, 8746

Siviero, A., Tamajo, E., Lutz, J., Wallerstein, G., \& ANS Collaboration 2012, Baltic Astron., 21, 188

Skopal, A. 1992, IBVS, 3780

Skopal, A. 2005, A\&A, 440, 995

Skopal, A. 2007, New Astron., 12, 597

Skopal, A., \& Pribulla, T. 2006, ATel, 882

Skopal, A., Vittone A. A., Errico, L., et al. 1997, MNRAS, 292, 703

Skopal, A., Vaňko, M., Pribulla, T., et al. 2007, Astron. Nachr., 328, 909

Skopal, A., Pribulla, T., Budaj J., et al. 2009, ApJ, 690, 1222

Skopal, A., Shugarov, S., Vaňko, M., et al. 2012, Astron. Nachr., 333, 242

Tomov, T., Munari, U., \& Marrese, P. M. 2000, A\&A, 354, L25 